\title{
Powder X-ray diffraction can differentiate between enantiomeric variants of calcium lactate pentahydrate crystal in cheese
}

\author{
G. F. Tansman, ${ }^{*}$ P. S. Kindstedt, ${ }^{* 1}$ and J. M. Hughes $†$ \\ *Department of Nutrition and Food Sciences, and \\ †Department of Geology, University of Vermont, Burlington 05405-0086
}

\section{ABSTRACT}

Powder X-ray diffraction has been used for decades to identify crystals of calcium lactate pentahydrate in Cheddar cheese. According to this method, diffraction patterns are generated from a powdered sample of the crystals and compared with reference cards within a database that contains the diffraction patterns of known crystals. During a preliminary study of crystals harvested from various Cheddar cheese samples, we observed 2 slightly different but distinct diffraction patterns that suggested that calcium lactate pentahydrate may be present in 2 different crystalline forms. We hypothesized that the 2 diffraction patterns corresponded to 2 enantiomeric forms of calcium lactate pentahydrate (L- and DL-) that are believed to occur in Cheddar cheese, based on previous studies involving enzymatic analyses of the lactate enantiomers in crystals obtained from Cheddar cheeses. However, the powder $\mathrm{X}$-ray diffraction database currently contains only one reference diffraction card under the title "calcium lactate pentahydrate." To resolve this apparent gap in the powder X-ray diffraction database, we generated diffraction patterns from reagent-grade calcium L-lactate pentahydrate and laboratory-synthesized calcium DLlactate pentahydrate. From the resulting diffraction patterns we determined that the existing reference diffraction card corresponds to calcium DL-lactate pentahydrate and that the other form of calcium lactate pentahydrate observed in cheese crystals corresponds to calcium L-lactate pentahydrate. Therefore, this report presents detailed data from the 2 diffraction patterns, which may be used to prepare 2 reference diffraction cards that differentiate calcium L-lactate pentahydrate from calcium DL-lactate pentahydrate. Furthermore, we collected crystals from the exteriors and interiors of Cheddar cheeses to demonstrate the ability of powder $\mathrm{X}$-ray diffraction to differentiate between the 2 forms of calcium lactate pentahydrate crystals in Cheddar

Received April 24, 2014.

Accepted September 15, 2014.

${ }^{1}$ Corresponding author: paul.kindstedt@uvm.edu cheeses. Powder X-ray diffraction results were validated using enzymatic assays for lactate enantiomers. These results demonstrated that powder X-ray diffraction can be used as a diagnostic tool to quickly identify different forms of calcium lactate pentahydrate that may occur in Cheddar cheese.

Key words: calcium lactate, crystal, X-ray diffraction, Cheddar, defect

\section{INTRODUCTION}

Powder X-ray diffraction (PXRD) has been used for decades to identify crystalline species that appear in aged cheeses. Cheese-crystal species include calcium phosphate, cysteine, calcium lactate pentahydrate (CLP), and tyrosine (Harper et al., 1953; Conochie et al., 1960; Pearce et al., 1973). Much of the research in the past has focused on the appearance of calcium lactate deposits on the surface of rindless Cheddar cheese, which is seen as a defect (Dybing et al., 1988; Johnson et al., 1990a; Swearingen et al., 2004).

While exploring the capabilities of PXRD to differentiate and identify crystals in cheese, we recently observed that crystals isolated from the surfaces of Cheddar cheese samples variably produced 1 of 2 slightly different but distinct diffraction patterns. One of the diffraction patterns could be matched to a reference card labeled "calcium lactate pentahydrate" [International Centre for Diffraction Data (ICDD) card number: 00-029-1596], whereas the other diffraction pattern could not be matched to any reference card and was regarded as an unidentified crystal. The discovery of an unidentified crystal in Cheddar cheese is noteworthy given that the diffraction patterns produced by approximately 250,000 known crystalline species have been identified and archived as reference cards in the PXRD instrumentation database.

Johnson et al. (1990b) showed that 2 types of CLP crystals form on Cheddar cheese depending on the enantiomeric form of lactate in the cheese serum. Although L-lactate is produced by the starter culture, which gives rise to crystals of calcium L-lactate pentahydrate (LCLP), it can be converted to D-lactate by nonstarter bacteria. Conversion of as little as $25 \%$ of L-lactate to 
D-lactate can cause all surface crystallization to occur as calcium DL-lactate pentahydrate (DL-CLP). Furthermore, Johnson et al. (1990b) demonstrated that DL-CLP contains equal quantities of D-lactate and Llactate. This suggests that in the presence of D-lactate and L-lactate, calcium will preferentially bond with one L-lactate and one D-lactate molecule, rather than with 2 L-lactate or 2 D-lactate molecules. Furthermore, Cao et al. (2001) demonstrated that DL-CLP is less soluble than L-CLP.

It is thus evident that 2 forms of CLP that have different formation dynamics and different solubilities can exist in Cheddar cheese. These characteristics suggest that the 2 forms of CLP also have different bonding structures and, thus, different crystal structures. Such a distinction, if it exists, can be observed as distinguishable PXRD patterns. However, as noted earlier, the PXRD database contains only one reference card for CLP of unspecified enantiomeric conformation, thus revealing a gap in the current database with respect to CLP.

Preliminary work was conducted to determine the enantiomeric identity of the CLP species designated by the reference card. These preliminary data indicated that reagent-grade L-CLP did not match the reference card. Calcium DL-lactate pentahydrate was not available through commercial suppliers. The following study was intended to determine whether the diffraction pattern in the ICDD reference card is indeed DL-CLP.

Calcium DL-lactate pentahydrate and L-CLP emerge in Cheddar cheese under different microbiological conditions (Johnson et al., 1990b; Chou et al., 2003; Agarwal et al., 2008). Therefore, the specific enantiomeric form of CLP in cheese represents useful diagnostic information. It is important for cheese researchers and cheesemakers to properly identify the enantiomeric form of unwanted CLP crystals when they occur so that they can address the underlying causes of the crystal formation, which may differ depending on the CLP variant. We hypothesize that PXRD can rapidly and reliably differentiate between enantiomeric variants of CLP crystals that form in cheese. The specific aims of the present study were to demonstrate that PXRD patterns of L-CLP and DL-CLP are distinguishable and to further demonstrate that PXRD can be used to distinguish between L-CLP and DL-CLP crystals obtained from Cheddar cheese.

\section{MATERIALS AND METHODS}

\section{Synthesis of DL-CLP Crystals}

A reagent-grade source of DL-CLP was not available. Therefore, we synthesized DL-CLP from reagent-grade
DL-lactic acid (Sigma-Aldrich Co. LLC, St. Louis, MO) and reagent-grade calcium carbonate (Sigma-Aldrich Co. LLC) using the following method.

Four grams of calcium carbonate was dissolved in 50 $\mathrm{mL}$ of deionized water. A total of $10 \mathrm{~mL}$ of DL-lactic acid was added slowly so that the effervescence would not overflow the $100-\mathrm{mL}$ beaker that served as the reaction vessel. The solution was stirred slowly with a magnetic stirrer until all of the effervescence had ceased and the solution was clear. A metal spatula was used to scratch the bottom of the glass beaker, which facilitated the crystallization of DL-CLP. The magnetic stirring was maintained until the solution became a semisolid.

The semisolid mixture of crystalline DL-CLP and solution was transferred to a Buchner funnel with \#541 filter paper (Sigma-Aldrich Co. LLC) fitted onto an aspirated vacuum flask and dried with a vacuum of residual solution. After no more solution was drawn from the sample for at least half an hour, the crystalline DL-CLP was transferred to a desiccator to further dry for $24 \mathrm{~h}$.

After drying in the desiccator, the sample was finely ground using a mortar and pestle and placed in an oven at $38^{\circ} \mathrm{C}$ for $12 \mathrm{~h}$. Because DL-CLP is hygroscopic (Sakata et al., 2005), this was intended to completely dry the sample. Care was taken not to drive off the associated water, which would skew the results of the experiment. When associated water molecules are evolved by either heating or high vacuum, CLP will lose its crystallinity, as indicated in the X-ray diffractograms. Thus, after each sample was dried, an X-ray diffraction pattern was generated to ensure that the water of hydration was still present. Those samples that were excessively dried to the point of becoming partially or completely anhydrous were discarded.

Approximately $0.8 \mathrm{~g}$ of dried sample was dissolved in $500 \mathrm{~mL}$ of deionized water as a stock solution for compositional analysis. Composition analyses of Dlactate, L-lactate, and calcium were then conducted as described below to ensure that the sample contained pure calcium DL-lactate pentahydrate.

\section{CLP Crystals from Cheese}

Several samples of experimentally produced unrinded Cheddar were obtained from a previous aging study. These samples displayed heavy surface crystallization. Two samples, each displaying a different diffraction pattern from its surface crystals, were eventually selected for further analysis. Calcium lactate precipitates were scraped from the surface of the cheese with a metal spatula. In addition, large granular crystals were found inside 3 blocks of commercially produced Vermont Cheddar. These crystals were excised using a spatula or 
dissection needle. Granular crystals from each block all displayed identical diffraction patterns, and the excised crystals were pooled.

Most of the collected crystalline material was defatted and dried using a single acetone wash in a Buchner funnel; a portion of the calcium lactate that was not washed in acetone was used to verify that the acetone wash did not create any diffraction artifacts. Diffraction patterns of defatted and non-defatted surface precipitates were compared; the acetone wash was expected to lower the baseline caused by noncrystalline phases, but care was taken to ensure that the acetone wash did not cause any crystallization of entities that would be detected on the diffractogram.

Once it was determined that the acetone wash was suitable for preparing surface precipitates for PXRD, only the dried and defatted sample powders were used for further analysis. Defatted samples provided less noisy diffractograms and were easier to work with when conducting chemical analysis.

Solutions of defatted surface crystals were dissolved in deionized water at the rate of approximately 0.04 $\mathrm{g}$ per $100 \mathrm{~mL}$ of $\mathrm{H}_{2} \mathrm{O}$ for chemical analysis. Solutions were shaken vigorously until almost all material was dissolved. After 15 min of shaking, any remaining solid material in solution was removed by filtration through Whatman \#1 filter paper (GE Healthcare Bio-Sciences, Pittsburgh, PA) and a Buchner funnel.

\section{Compositional Analysis of CLP Crystals}

For the analysis of synthesized DL-CLP, a 10-mL aliquot was taken from the stock solution and diluted to $100 \mathrm{~mL}$; a $50-\mathrm{mL}$ aliquot of this solution was then diluted to $100 \mathrm{~mL}$ to yield a solution that contained approximately $0.04 \mathrm{~g}$ of DL-CLP per $100 \mathrm{~mL}$ of deionized water. These steps were necessary to achieve a lactate concentration that was appropriate for the enzymatic lactate assay (test kit no. 11112821035, Boehringer Mannheim/R-Biopharm AG, Darmstadt, Germany) used to quantify lactate enantiomers in solution. Aliquots of $0.1 \mathrm{~mL}$ were tested in paired triplicate for $\mathrm{D}$ lactate and L-lactate according to the manufacturer's directions, in which the concentrations of both D- and L-lactate are measured sequentially for each aliquot. Enzymatic reactions were allowed to proceed at ambient temperature until absorbance values, measured at $340 \mathrm{~nm}$ using a Thermo Scientific Genesys 10S UVVIS Spectrophotometer (Thermo Fisher Scientific Inc., Waltham, MA), ceased rising.

Aliquots $(10 \mathrm{~mL})$ of the stock solution were analyzed in triplicate for calcium. Standard EDTA solution (Fisher Scientific, Pittsburgh, PA) and murexide indicator (Fisher Scientific) were used in the titration. The aliquots were transferred quantitatively to a beaker using deionized water and slightly alkalized with 5 drops of $0.1 \mathrm{M} \mathrm{NaOH}$ (Fisher Scientific). A spatula-tip-full of murexide (approximately $0.02 \mathrm{~g}$ ) was added to the beaker. Ethylenediaminotetraacetate was added to the beaker from a burette until a lingering color change was observed.

For the analysis of CLP crystals from cheese, 0.1$\mathrm{mL}$ aliquots were taken from a stock solution, and the enzymatic assay for L and DL lactic acid (test kit no. 11112821035, Boehringer Mannheim/R-Biopharm AG) was conducted according to the directions. Enzymatic reactions were allowed to proceed at ambient temperature until absorbance values, measured at $340 \mathrm{~nm}$ using a Thermo Scientific Genesys 10S UV-VIS Spectrophotometer (Thermo Fisher Scientific Inc.), ceased rising, which often took longer for cheese precipitates than for synthetic samples.

\section{Diffraction Pattern of CLP Crystals}

Diffraction patterns were generated from synthetic DL-CLP and cheese crystals using a MiniFlex II powder X-Ray diffractometer (Rigaku, The Woodlands, TX) and analyzed to verify the crystallinity and purity of the synthetic sample and identify cheese crystals.

Powdered samples were finely ground using a mortar and pestle and transferred to a glass diffraction slide with a well. The powder in the slide well was leveled with the surface of the diffraction slide using a microscope slide.

Diffractograms were generated at a speed of $2^{\circ} 2 \theta /$ min between 5 and $50^{\circ} 2 \theta$. Diffraction patterns were compared with the existing card (ICDD card number: 00-029-1596) titled "calcium lactate pentahydrate" using a specialized calculation called a figure of merit. The figure of merit was calculated automatically by PDXL, the diffractometer software package (Rigaku). An acceptance threshold of one was set for the figure of merit.

\section{Statistical Analysis}

Mean concentrations of L-lactate, D-lactate, and calcium in synthesized DL-CLP were compared with the theoretical values using 2 -tailed $t$-tests with $\alpha=0.05$. Theoretical values were calculated using molar equivalent weights for CLP-containing calcium, D-lactate, L-lactate, and water in a 1:1:1:5 molar ratio.

\section{RESULTS}

\section{Characterization of Synthetic DL-CLP}

The measured means for D-lactate, L-lactate, and calcium in the synthesized DL-CLP are compared with 
Table 1. Theoretical and measured values for the composition of laboratory-synthesized calcium DL-lactate pentahydrate ${ }^{1}$

\begin{tabular}{lcccc}
\hline & & \multicolumn{3}{c}{ Measured } \\
\cline { 3 - 5 } Component & Theoretical $^{2}$ & Mean & SD & CV \\
\hline L-Lactate (\%) & 29.23 & 29.19 & 0.003 & 0.01 \\
D-Lactate (\%) & 29.23 & 29.50 & 0.005 & 0.02 \\
Calcium (\%) & 13.00 & 12.90 & 0.001 & 0.01 \\
Water $^{3}(\%)$ & 28.54 & 28.11 & 0.380 & 1.36 \\
\hline
\end{tabular}

${ }^{1}$ All measured means were not different from theoretical values $(P>$ $0.05)$.

${ }^{2}$ Theoretical values were calculated using molar equivalent weights for calcium lactate pentahydrate containing calcium, D-lactate, L-lactate, and water in a 1:1:1:5 molar ratio.

${ }^{3}$ Values for water were determined by difference (not measured): $100 \%$ - (\% L-lactate $+\%$ D-lactate $+\%$ calcium $)$.

the theoretical values in Table 1 . The water content, calculated by difference $[100 \%-(\%$ L-lactate $+\%$ Dlactate $+\%$ calcium)], is also compared with the theoretical value. Measured means for D-lactate, L-lactate, and calcium did not differ significantly $(P>0.05)$ from the theoretical values. The data confirm that the synthetic crystalline material consisted of DL-CLP.

The diffraction pattern generated from the synthetic DL-CLP is shown in Figure 1. The bars represent the reference card (ICDD card number: 00-029-1596) labeled "calcium lactate pentahydrate." Note that the reference bars match the diffraction peaks and that the baseline of the diffraction is very close to zero. This, along with the tests for lactate enantiomers and calcium (Table 1 ), indicates that the synthetic DL-CLP was very pure. Most importantly, this clearly demonstrates that the pattern in the diffractometer reference card titled "cal- cium lactate pentahydrate" can be characterized more specifically as calcium DL-lactate pentahydrate.

The reference card titled "calcium lactate pentahydrate" was originally published by Folen (1975) and was intended for the study of illicit drugs, in which CLP is occasionally used as a filler material. The author presents metrics for diffraction peak intensity and angle but does not specify the source or specific chemical makeup of the "reagent-grade chemicals" that were used to produce this specific diffraction reference card. Since 1975 several papers have been published in the field of cheese science alone that used X-ray diffraction to study CLP (Dybing et al., 1988; Chou et al., 2003; Agarwal et al., 2006). Nonetheless, the subtle differences between the diffraction patterns of DL-CLP and L-CLP were completely overlooked during that time.

Figure 2, which compares the diffraction pattern of reagent-grade L-CLP and the card titled "calcium lactate pentahydrate," reveals substantial differences in diffraction peak locations along the x-axis. In particular, note the location in Figure 2 of the second large peak, at approximately 9 degrees, and the 2 smaller peaks at approximately 10 and 15 degrees; none of these peaks fit the reference card as well as the corresponding peaks in Figure 1.

The 2 diffraction patterns in Figures 1 and 2 do have a striking similarity that may partially explain why the diffraction pattern for L-CLP was never characterized. In fact, even using the PXRD-specific metric, the figure of merit, may fail to distinguish between these 2 diffraction patterns unless the threshold of distinction is kept conservatively low. The corresponding figure of merit for the diffraction-reference card pair in Figure 1 is

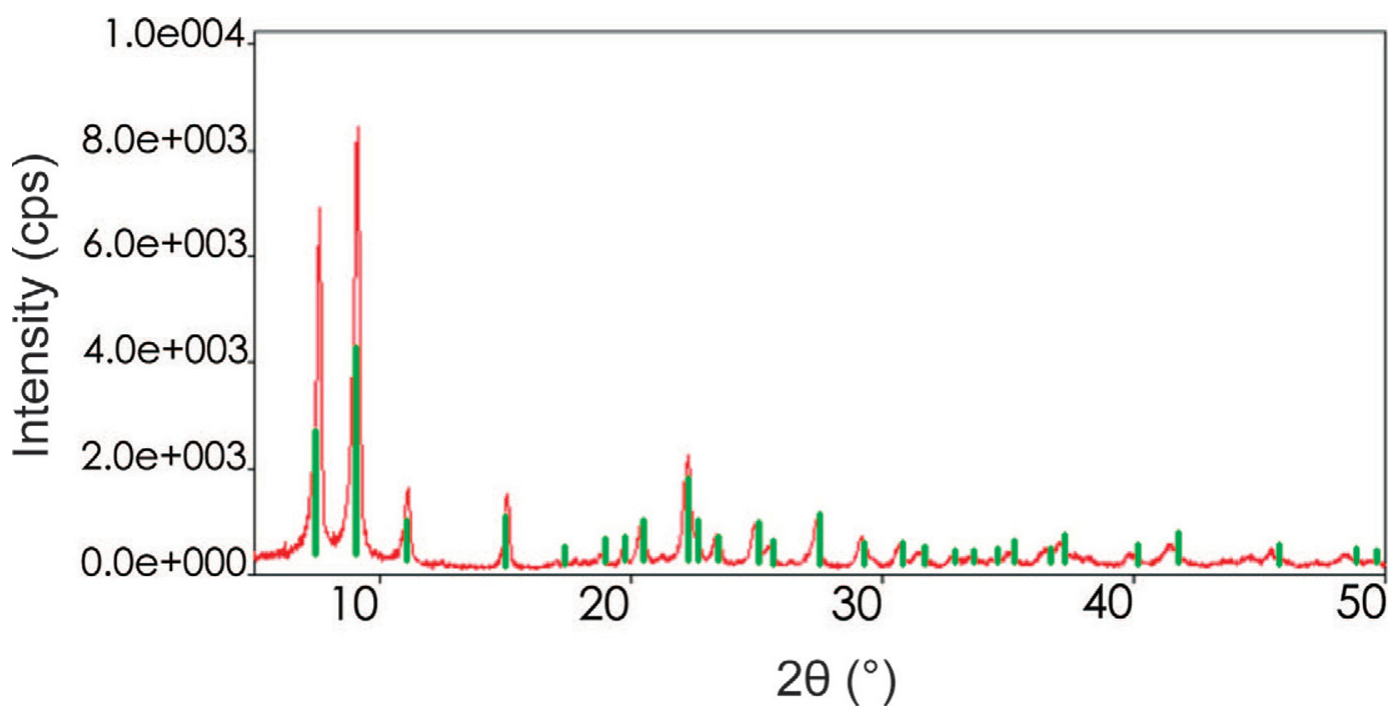

Figure 1. Diffraction intensities (in cps) by diffraction angle in degrees $2 \theta$ for laboratory-synthesized calcium DL-lactate pentahydrate. The green bars represent the reference card (ICDD card number: 00-029-1596) labeled "calcium lactate pentahydrate." 


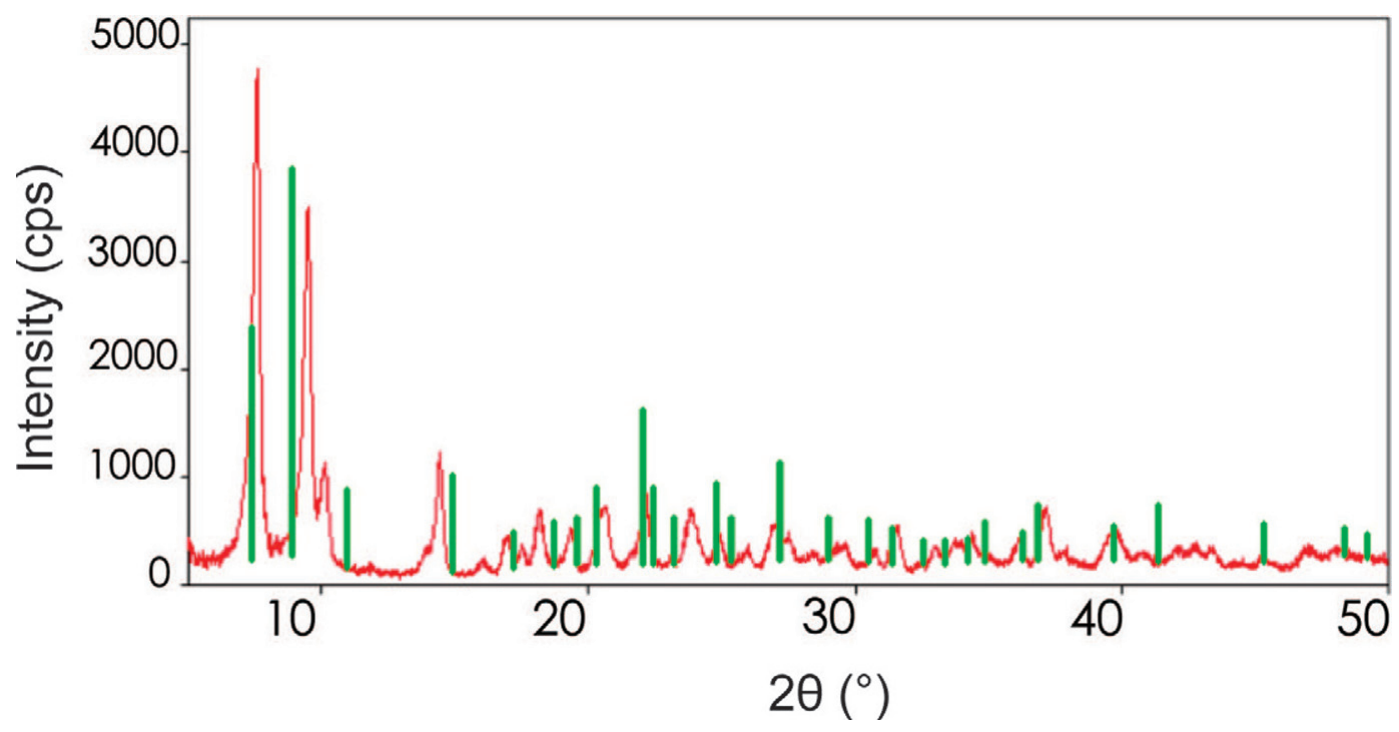

Figure 2. Diffraction intensities (in cps) by diffraction angle in degrees $2 \theta$ for reagent-grade calcium L-lactate pentahydrate. The green bars represent the reference card (ICDD card number: 00-029-1596) labeled "calcium lactate pentahydrate."

below 0.839 , whereas the figure of merit for the diffraction-reference pair in Figure 2 is above 1.651. These figure-of-merit values are close, yet they are precise to about \pm 0.2 , depending on the purity and quality of the sample, and can be used to distinguish the 2 diffraction patterns.

\section{New Reference Card for L-CLP}

A new reference card titled "calcium L-lactate pentahydrate," based on the diffraction pattern generated from the reagent-grade L-CLP (Figure 2), is thus proposed. In addition, the existing reference card (ICDD card number: 00-029-1596), originally published by Folen (1975), should be renamed "calcium DL-lactate pentahydrate" to reflect its identity more precisely. For this purpose, Tables 2 and 3 present more detailed metrics from Figures 1 and 2, respectively.

\section{Characterization of CLP Crystals from Cheese}

Diffraction patterns were generated from crystals isolated from several different Cheddar cheese samples. Two experimentally produced cheeses were selected for further analysis; each cheese had heavy surface crystallization. One cheese had crystals that diffracted as DL-CLP, and the other had surface crystals that diffracted as L-CLP. Each crystalline sample was analyzed enzymatically for D- and L-lactate enantiomers. In addition, several granular crystals from the interior of commercially produced Vermont Cheddar were similarly analyzed.
Crystalline material that produced a DL-CLP diffraction pattern consistently yielded equivalent quantities of D- and L-lactate by enzymatic analysis, as expected. Furthermore, crystalline material that produced L-CLP diffraction patterns consistently yielded only L-lactate by enzymatic analysis, thus confirming the capacity of

Table 2. Tabulated peak angles, D-values, and heights for calcium DL-lactate pentahydrate ${ }^{1}$

\begin{tabular}{lrr}
\hline $2 \theta\left(^{\circ}\right)$ & $\mathrm{D}(\mathrm{A})$ & Height $(\mathrm{cps})$ \\
\hline $7.581(5)$ & $11.652(7)$ & $4,252(84)$ \\
$9.090(4)$ & $9.720(4)$ & $5,316(94)$ \\
$11.117(9)$ & $7.952(6)$ & $833(37)$ \\
$15.082(6)$ & $5.870(2)$ & $932(39)$ \\
$18.88(4)$ & $4.697(9)$ & $121(14)$ \\
$19.6(2)$ & $4.54(5)$ & $27(7)$ \\
$20.389(15)$ & $4.352(3)$ & $456(28)$ \\
$22.230(6)$ & $3.9956(11)$ & $1,265(46)$ \\
$22.653(15)$ & $3.922(2)$ & $351(24)$ \\
$23.429(13)$ & $3.794(2)$ & $306(23)$ \\
$24.922(12)$ & $3.5699(16)$ & $470(28)$ \\
$25.54(2)$ & $3.485(3)$ & $190(18)$ \\
$27.353(11)$ & $3.2578(13)$ & $563(31)$ \\
$29.10(3)$ & $3.066(3)$ & $311(23)$ \\
$30.57(4)$ & $2.922(4)$ & $152(16)$ \\
$31.59(3)$ & $2.830(2)$ & $92(12)$ \\
$33.64(12)$ & $2.662(9)$ & $57(10)$ \\
$35.09(2)$ & $2.5555(15)$ & $114(14)$ \\
$36.334(19)$ & $2.4706(13)$ & $131(15)$ \\
$36.96(3)$ & $2.4301(16)$ & $140(15)$ \\
$39.70(3)$ & $2.2686(17)$ & $118(14)$ \\
$41.41(2)$ & $2.1787(13)$ & $210(19)$ \\
$44.54(7)$ & $2.033(3)$ & $79(12)$ \\
$45.32(3)$ & $1.9996(12)$ & $149(16)$ \\
$48.34(6)$ & $1.881(2)$ & $92(12)$ \\
\hline
\end{tabular}

${ }^{1}$ Numbers in parentheses are $1 \mathrm{SD}$ of the least units cited. 
Table 3. Tabulated peak angles, D-values, and heights for calcium L-lactate pentahydrate ${ }^{1}$

\begin{tabular}{lrr}
\hline $2 \theta\left(^{\circ}\right)$ & $\mathrm{D}(\mathrm{A})$ & Height $(\mathrm{cps})$ \\
\hline $7.595(8)$ & $11.630(12)$ & $2,710(67)$ \\
$9.483(7)$ & $9.319(7)$ & $2,009(58)$ \\
$10.134(15)$ & $8.722(13)$ & $558(31)$ \\
$14.401(11)$ & $6.146(5)$ & $702(34)$ \\
$16.93(2)$ & $5.234(7)$ & $193(18)$ \\
$17.52(3)$ & $5.057(9)$ & $112(14)$ \\
$18.143(17)$ & $4.886(4)$ & $326(23)$ \\
$19.372(19)$ & $4.578(4)$ & $203(18)$ \\
$20.559(17)$ & $4.316(4)$ & $368(25)$ \\
$22.130(15)$ & $4.014(3)$ & $393(26)$ \\
$23.801(18)$ & $3.735(3)$ & $311(23)$ \\
$24.89(2)$ & $3.575(3)$ & $184(17)$ \\
$26.96(3)$ & $3.304(4)$ & $205(18)$ \\
$27.47(5)$ & $3.244(6)$ & $128(15)$ \\
$29.65(6)$ & $3.010(6)$ & $83(12)$ \\
$31.54(3)$ & $2.834(3)$ & $233(20)$ \\
$32.97(6)$ & $2.715(5)$ & $104(13)$ \\
$33.78(10)$ & $2.651(7)$ & $104(13)$ \\
$34.38(8)$ & $2.606(6)$ & $98(13)$ \\
$35.93(12)$ & $2.497(8)$ & $27(7)$ \\
$37.10(2)$ & $2.4212(15)$ & $317(23)$ \\
$39.788(15)$ & $2.2637(8)$ & $179(17)$ \\
$43.18(11)$ & $2.093(5)$ & $76(11)$ \\
\hline
\end{tabular}

${ }^{1}$ Numbers in parentheses are $1 \mathrm{SD}$ of the least units cited.

PXRD to distinguish between the 2 CLP enantiomeric variants in cheese. Whereas crystals of L-CLP were likely caused by L-lactate produced by starter culture, DL-CLP crystallization was probably triggered by the conversion of L- to D-lactate by nonstarter bacteria during ripening (Johnson et al., 1990b; Chou et al., 2003; Agarwal et al., 2008).

Surface crystalline deposits were variably DL-CLP and L-CLP, whereas the large interior crystals were exclusively DL-CLP. Notably, each cheese sample contained only one enantiomeric form of CLP, regardless of the extent of crystallization, which is consistent with the view that in the presence of D-lactate, calcium preferentially bonds with one D-lactate and one L-lactate molecule, rather than 2 L-lactate molecules.

No obvious morphological differences were observed visually between surface crystalline deposits of DLCLP and those of L-CLP. However, the large spheroid crystals of DL-CLP that formed within the interior of some cheese samples were much harder than any of the surface deposits. These interior crystals appeared to form preferentially within curd junctions and mechanical openings, which may provide nucleation sites for crystallization. Very large interior crystals are a cause of significant concern among some manufacturers of long-aged Cheddar cheese, based on anecdotal reports that our laboratory has received from industry sources. Thus, further work to identify the nonstarter bacteria responsible for the production of the D-lactate that gives rise to such crystals, as well as microstruc- tural and macrostructural factors that contribute to the formation of very large interior crystals of DL-CLP, appears warranted.

\section{DISCUSSION}

\section{PXRD to Determine the Degree of Dehydration of Laboratory-Synthesized CLP}

As mentioned earlier, the laboratory-synthesized DL-CLP needed to be dried of hygroscopic water without driving off the water associated with the crystal structure. According to Sakata et al. (2005), anhydrous CLP does not diffract X-rays and will instead display a characteristic halo pattern. Using this as a guide, diffraction patterns from oven- and vacuum-dried samples were produced to ensure that associated hydration water was not driven off.

Figure 3 depicts the diffraction pattern from a sample of DL-CLP that was oven dried at $80^{\circ} \mathrm{C}$ for several hours. Note the complete lack of characteristic diffraction peaks and the broad amorphous humps that typify poorly crystallized material; this indicates that the sample was excessively dried to the point of chemical dehydration. Figure 4 depicts the diffraction pattern from a sample of DL-CLP that was dried under high vacuum at ambient temperature for $48 \mathrm{~h}$. Several of the larger characteristic peaks can still be distinguished, but the raised baseline and severely broadened peaks indicate a partial loss of crystallinity. This last point demonstrates the sensitivity of DL-CLP to various drying treatments and the trial and error that was necessary to find an appropriate drying method.

\section{Synthesis of a New CLP Variant}

It should be noted that while synthesizing a sample of DL-CLP, a crystalline variant of CLP was produced as a major impurity. This can be seen in the diffraction of that sample in Figure 5. The diffraction pattern of DL-CLP, as indicated by the bars of the reference card, can be clearly identified; however, large unexplained peaks also appear. No card exists in the database that satisfactorily identifies the crystalline species that is responsible for the extra peaks, but based on circumstance, it is likely caused by a hydration variant of calcium lactate.

Hydration variants, monohydrate and trihydrate, have been noted in the literature (Sakata et al., 2005; Peveler and Palmer, 2012), but reference cards have not been published to date. The variant may be 1 of these 2 species, although identification of this crystalline species is beyond the scope of this publication. To avoid the synthesis of this contaminant, DL-CLP synthesis 


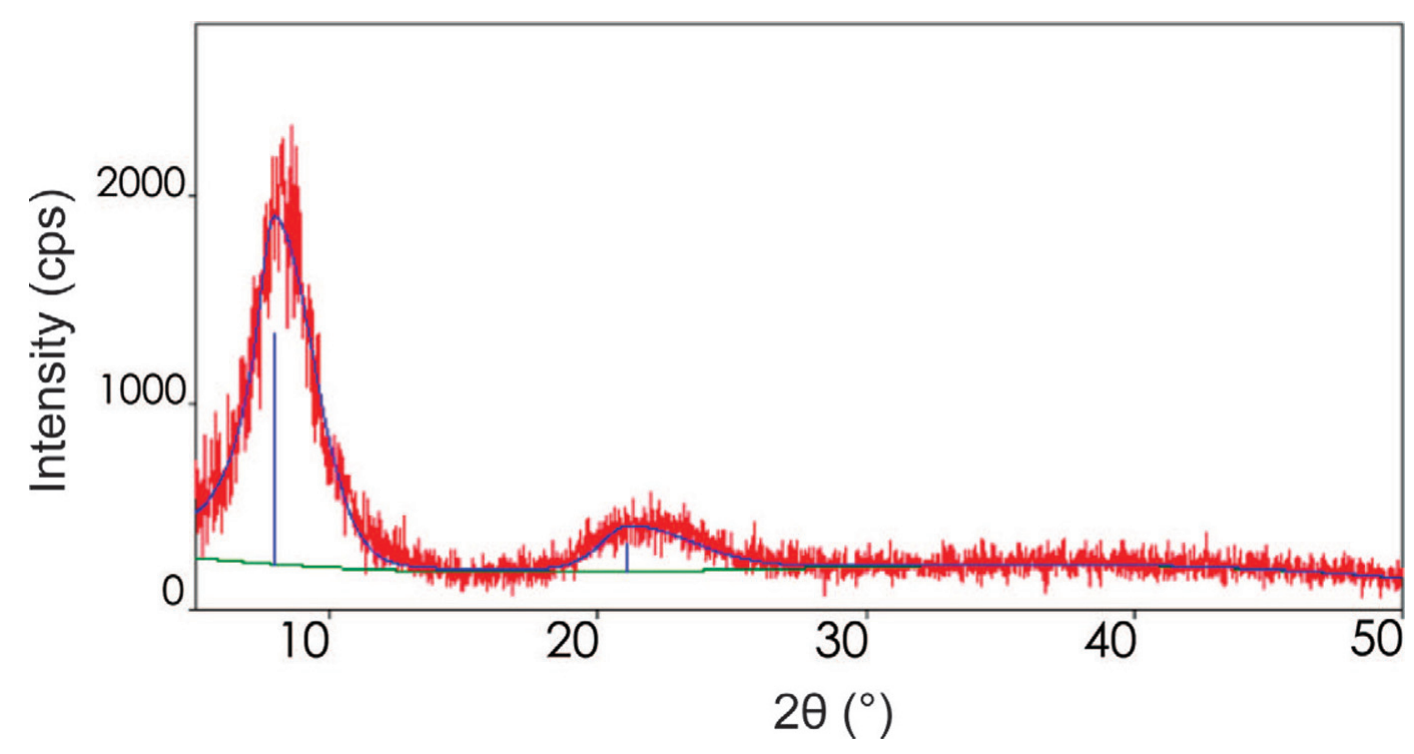

Figure 3. Characteristic halo pattern and absence of X-ray diffraction peaks from calcium DL-lactate pentahydrate that was oven dried at $80^{\circ} \mathrm{C}$ to the point of total dehydration. Color version available in the online PDF.

was not allowed to proceed to the point where water became a limiting reagent; this was relevant because only a fairly concentrated solution would yield crystals, but the synthesis of crystals also incorporates what water is available into the crystal structure.

The synthesis of a hydration variant along with DLCLP would have skewed the analysis of the synthesized powder because hydration variants do not weigh the same as DL-CLP. Thus, diffraction patterns were generated after each synthesis to ensure the absence of this contaminant and the purity of the sample.

\section{PXRD to Distinguish Between CLP Variants from Cheese Samples}

The ability to easily differentiate between DL-CLP and L-CLP is a valuable tool for cheese researchers who are supporting cheesemakers. Diffraction patterns can be generated within minutes and with almost no sample preparation. Diffractometers are relatively inexpensive to operate, are fairly common in universities, and are used in many different disciplines ranging from geology to pharmaceuticals. In contrast, enzymatic

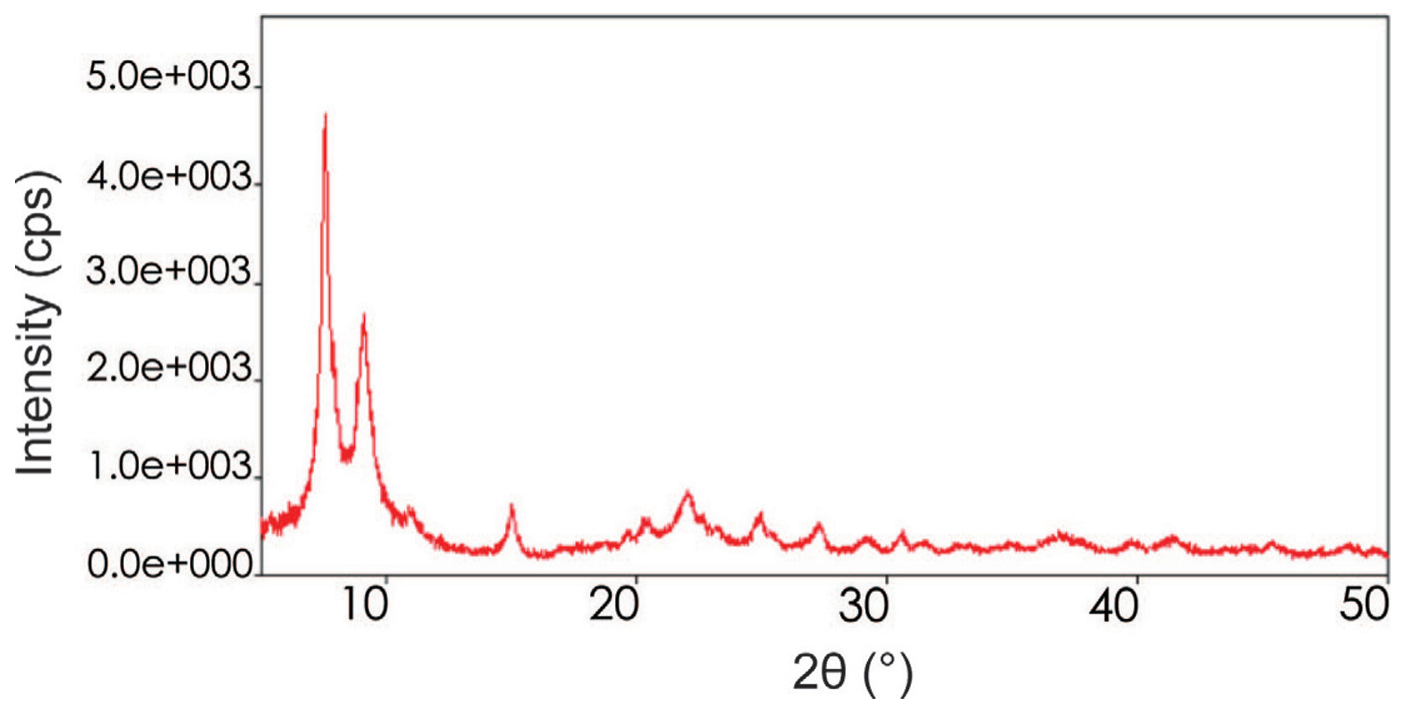

Figure 4. Partial loss of X-ray diffraction capability from calcium DL-lactate pentahydrate that was excessively dried under high vacuum at ambient temperatureColor version available in the online PDF. 


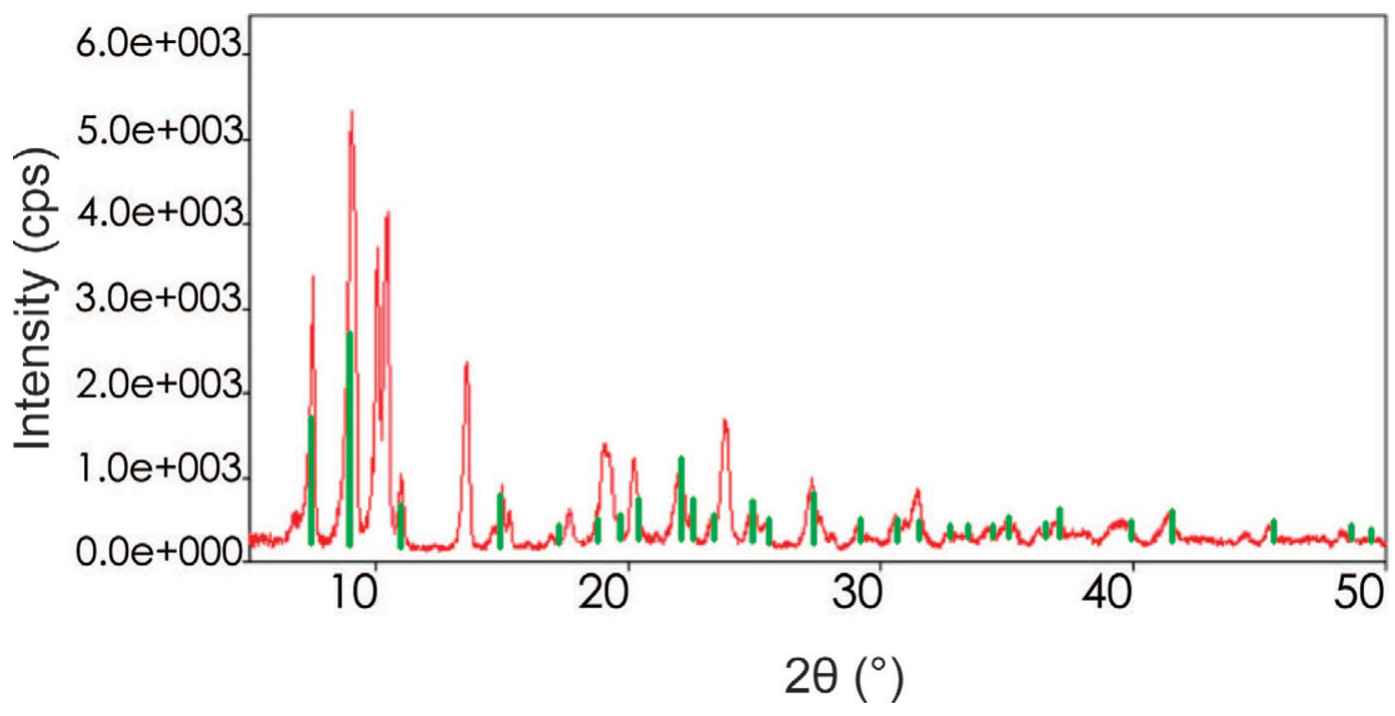

Figure 5. Diffraction pattern of calcium DL-lactate pentahydrate produced under conditions in which water was a limiting reagent; in addition to the characteristic peaks, indicated by the green bars of the reference card (ICDD card number: 00-029-1596) labeled "calcium lactate pentahydrate," extraneous peaks also appear.

analysis, which is the current practice (Severn et al., 1986; Agarwal et al., 2008), can take up to several hours, and assay kits have a limited shelf-life and are expensive. Furthermore, crystalline deposits in cheese contain varying quantities of noncrystalline material such as cheese serum, fat, and protein. To get accurate readings, the lactate content of the samples must be within a narrow range, which makes it difficult to predict the appropriate sample size when working with crystals that contain highly variable levels of noncrystalline material.

Using PXRD, any noncrystalline material will increase the background noise of the diffraction pattern, but the diffraction reading can be easily observed and analyzed even when noncrystalline material exists in excess. In addition, PXRD, by design, discriminates between crystalline CLP and free calcium, lactate, and water that may exist as noncrystalline impurities in the sample; chemical analysis does not have this capability.

Understanding the composition of unwanted crystalline deposits in and on cheese can be of great benefit to producers. It has previously been established that nonstarter bacteria can convert L-lactate to D-lactate, and in doing so decrease the solubility of CLP in the cheese serum (Johnson et al., 1990b; Chou et al., 2003; Agarwal et al., 2006). Differentiating between DL-CLP and L-CLP can give cheesemakers a much clearer snapshot of the chemical and microbiological environment in their cheeses. Depending on the type of CLP, different process changes can be proposed. For instance, if L-CLP forms, it indicates that racemizing nonstarter bacteria are likely absent but that soluble calcium and
L-lactate exist in excess. If DL-CLP forms, the microbiology of the cheese is a main cause, and various steps to standardize the microbiology may be proposed.

\section{CONCLUSIONS}

This study has demonstrated the ability of PXRD to rapidly and accurately differentiate between DL-CLP and L-CLP from synthetic sources and from Cheddar cheese. Where available, PXRD can thus replace timeconsuming and costly enzymatic assays for the analysis of crystalline deposits in and on cheese.

\section{ACKNOWLEDGMENTS}

The financial support of USDA Hatch Project VTH01905 is gratefully acknowledged. The National Science Foundation is gratefully acknowledged through support of grant EAR-0922961 for purchase of the Xray diffractometer.

\section{REFERENCES}

Agarwal, S., R. J. Powers, B. G. Swanson, S. Chen, and S. Clark. 2008. Influence of salt-to-moisture ratio on starter culture and calcium lactate formation. J. Dairy Sci. 91:2967-2980.

Agarwal, S., K. Sharma, B. G. Swanson, U. Yuksel, and S. Clark. 2006. Nonstarter lactic acid bacteria biofilms and calcium lactate crystals in Cheddar cheese. J. Dairy Sci. 89:1452-1466.

Cao, X., H. Lee, H. Yun, and Y. Koo. 2001. Solubilities of calcium and zinc lactate in water and water-ethanol mixture. Korean J. Chem. Eng. 18:133-135.

Chou, Y.-E., G. Edwards, L. O. Luedecke, M. P. Bates, and S. Clark. 2003. Nonstarter lactic acid bacteria and aging temperature affect calcium lactate crystallization in Cheddar cheese. J. Dairy Sci. $86: 2516-2524$. 
Conochie, J., J. Czulak, A. J. Lawrence, and W. F. Cole. 1960. Tyrosine and calcium lactate crystals on rindless cheese. Aust. J. Dairy Technol. 15:120.

Dybing, S. T., J. A. Wiegand, S. A. Brudvig, E. A. Huang, and R. C. Chandan. 1988. Effect of processing variables on the formation of calcium lactate crystals on Cheddar cheese. J. Dairy Sci. 71:1701-1710.

Folen, V. A. 1975. X-ray powder diffraction data for some drugs, excipients, and adulterants in illicit samples. J. Forensic Sci. 20:348-372.

Harper, W. J., A. M. Swanson, and H. H. Sommer. 1953. Observations on the chemical composition of white particles in several lots of Cheddar cheese. J. Dairy Sci. 36:368-372.

Johnson, M. E., B. A. Riesterer, C. Chen, B. Tricomi, and N. F. Olson. 1990a. Effect of packaging and storage conditions on calcium lactate crystallization on the surface of Cheddar cheese. J. Dairy Sci. 73:3033-3041.
Johnson, M. E., B. A. Riesterer, and N. F. Olson. 1990b. Influence of nonstarter bacteria on calcium lactate crystallization on the surface of Cheddar cheese. J. Dairy Sci. 73:1145-1149.

Pearce, K. N., L. K. Creamer, and J. Gilles. 1973. Calcium lactate deposits on rindless Cheddar cheese. N. Z. J. Dairy Sci. Tech. 8:3-7.

Peveler, W. W., and T. G. Palmer. 2012. Effects of magnesium lactate dihydrate and calcium lactate monohydrate on 20-km cycling time trial performance. J. Strength Cond. Res. 26:1149-1153.

Sakata, Y., S. Shiraishi, and M. Otsuka. 2005. Characterization of dehydration and hydration behavior of calcium lactate pentahydrate and its anhydrate. Colloids Surf. B Biointerfaces 46:135-141.

Severn, D. J., M. E. Johnson, and N. F. Olson. 1986. Determination of lactic acid in Cheddar cheese and calcium lactate crystals. J. Dairy Sci. 69:2027-2030.

Swearingen, P. A., D. E. Adams, and T. L. Lensmire. 2004. Factors affecting calcium lactate and liquid expulsion defects in Cheddar cheese. J. Dairy Sci. 87:574-582. 